http://jmscr.igmpublication.org/home/

ISSN (e)-2347-176x ISSN (p) 2455-0450

crossref DOI: https://dx.doi.org/10.18535/jmscr/v8i7.47

Journal Of Medical Science And Clinical Research

\title{
The relation between the tracking/reporting system for MSLs in medical affairs departments in pharmaceutical companies located within the MENA region and its impact on the MSL productivity
}

\author{
Authors \\ S. Ibrahim ${ }^{1}$, A. El Said ${ }^{2}$ and A. Elgouhary ${ }^{3}$ \\ ${ }^{1}$ B Pharm; MBA - Amgen Cairo, Egypt \\ ${ }^{2}$ Adjunct Professor, ESLSCA, Cairo, Egypt \\ ${ }^{3} \mathrm{MD}$ - Pfizer Vaccines Jeddah, Saudi Arabia \\ *Corresponding Author \\ S. Ibrahim
}

\begin{abstract}
Background: The Medical Science Liaison (MSL) who has a strong scientific and clinical background is the disease and drug expert within the medical (affairs) department of the pharmaceutical/biotech company. Doing the important role of being the first point-of-contact to answer any drug or disease related questions from the internal as well as external stakeholders. Previously, MSL initial function was to build rapport with KOLs, but recently, the MSL role has evolved during the past 20 years in many ways from being only a support for commercial teams to the forefront of pharmaceutical practice. The MSL role has become one of the most important customer focused roles within the pharmaceutical/biotech industry and this has led to a massive increase in the MSL work force globally.

The research objective is to explain the relation between the tracking/reporting system for MSLs in medical affairs departments in pharmaceutical companies located within the MENA region and its impact on the MSL productivity therefore impacting the growth rate of the company taking in consideration the varying usage of the systems, frequency of tracking \& choice of metrics trying to prove the direct relation between all the variables.

Methods: This research will be an exploratory research to test a non-directional hypothesis in this setting. The type of investigation is a descriptive analysis, correlation which will be non-controlled (amount of control will be minimal $(0-20 \%))$. The setting will be a field study and the time horizon will be cross-sectional with regards to the theoretical framework highlighted below.

Data collection will be done through an electronic questionnaire developed through Free-online Surveys ${ }^{\circledR}$ to be distributed to a sample of 73 MSLs from the population of MSLs in pharmaceutical companies located in the MENA Region during a time span from 2010 till 2019.

Results: The list of 73 targeted respondents was made up of $53 \%$ Male and $47 \%$ Female. $37 \%$ of the respondents were currently working as MSLs while the remaining $63 \%$ previously worked as MSLs.

Metrics captured by these systems were $90 \%$ a mixture of quantitative as well as qualitative metrics. $43 \%$ of these metrics were company customized, 33\% were general or generic metrics and $29 \%$ were therapy area customized. The tracking of the metrics was done monthly in 67\% of the cases, followed by quarterly in 34\%, annually in $24 \%$ with a clear overlap between most companies in monthly and quarterly tracking.

$33 \%$ agreed that MSL performance reports generated by the systems, should be readily made available to cross-functional stakeholders and $47 \%$ agreed that the use of these systems increased their productivity.

Conclusion: The significant majority of MSL respondents believe that the use of tracking and reporting systems will increase their productivity and nearly half of the respondents believe that making the generated MSL reports readily available for internal stakeholders will further enhance productivity while not negatively impacting motivation of MSLs in pharmaceutical companies operating in the MENA Region.
\end{abstract}




\section{Problem Definition Contextual Factors \\ Background: MSL Rolein-Depth}

The MSL who has a strong scientific and clinical background is doing the important role of being the first point-of-contact to answer any drug or disease related questions from the internal as well as external stakeholders. These external stakeholders could include a wide spectrum of HCPs in the medical society.

MSLs must therefore be excellent communicators (Bijker, 2017). MSLs mainly are minded with medical affairs work in the pre-approval stage of the drug - i.e. the drug is not allowed to be sold yet - or post-approval of the drug - a sales team is present to sell the drug - where the role substantially changes compared to when you are working on a pipeline drug (Bijker, 2017).

MSL work often includes close involvement in clinical trials medical support as well as discussions with HCPs - mainly KOLs - in light of internal stakeholder questions on various topics around: the future clinical development of the drug (in new indications); registration and reimbursement of the drug; gaps in medical education of the general clinicians on the disease; and competitive (dis) advantages of the drugs, for example. These discussions will generate crucial insights which the MSL brings back to internal stakeholders to help them formulate better strategies that will harness clinical success for the patient, the treating doctor and the company (Bijker, 2017). MSL role has evolved and is challenged by the large amount of data they share with many internal \& external stakeholders while ensuring it is accurate, updated and complies with local regulations. This has caused the MSL to be a central hub of information that needs to be managed efficiently since the role of the MSL has expanded to include a vast array of technical \& analytical skills that must be met by a set of updated, effective enablers to help them do their job more efficiently.

\section{Business Anatomy (The Nine Elements)}

The MSL role has become one of the most important customer focused roles within the pharmaceutical/biotech industry and this has led to a massive increase in the MSL workforce globally.

Medical Affairs not only has the sole responsibility of pre-launch activities, but now has the mandate for all medical activities related to marketed products and provides input to both clinical development and commercialization. MSLs play a unique and important role in being available to inform HCPs about current and emerging management solutions (prevention/treatment) in different therapeutic areas.

With increased emphasis on building and maintaining long-term relationships with KOLs, the MSL role must be based on fair metrics to evaluate and reward appropriately. Since the MSL role is scientific, it should include IITs, clinical trials, publications, speeches and scientific exchange as well as strategic associations.

Although there is no universal blueprint for MSL success, some best practices are emerging. An effective MSL strategy will segment MSL teams to address all key stakeholders that can affect a brand's success. MSLs have historically proven more valuable and effective during the pre-launch phase than in post-launch.

Measuring the value of MSLs will always involve a mix of quantitative and qualitative variables. A quantitative variable may be the amount of face time an MSL spends with a physician. A qualitative measure might be the medical insight derived from those conversations.

The currently used reporting systems are very basic and lack clear structure, focusing on a quantitative metrics, like number $\&$ duration of visits, to direct managers that are not made readily available to internal stakeholders overlooking the importance of the qualitative metrics.

Earlier on, the used reporting systems consisted 
of word documents and excel sheets and only recently have web-based CRM tools started emerging in the MENA Region. These systems, besides also not having clear structure, are under-utilized.

The main management function subject of this research is organizing and controlling since the proposed problem discusses the drawbacks of having a poor tracking and reporting system.

It is targeted towards the middle managers of the chain of responsibility of these organizations as the MSL and their direct managers or field heads as well as their senior superiors either medical advisors or medical managers who base their work and strategies on the efficient outcome of the MSL interactions.

The business function subject to discussion here is experience/knowledge as well as HR and IT. Experience/knowledge will be focusing on the maturity level of the associates to understand the importance of performance management metrics reporting systems and effectively using them. IT will primarily focus on the new trends of CRM \& clouded services that provide digital solutions to reporting systems and real-time dashboards for better planning, organizing and controlling which will impact HR in optimizing MSL teams in these organizations.

The internal environment will include the MSL internal customers/stakeholders like medical advisors, medical managers or commercial colleagues as well as the employees they interact with internally to perform their reporting tasks like IT \& share point administrators.

The time frame of this research will be to provide prospective conclusions on the observations made to the subject of matter from 2010 till 2019 since this is the timeframe that included the highest transformation and advancement of the MSL role and technological tools.

The external environment surrounding the MSL is dynamic and has changed a lot within the last 20 years. Some changes specific to the MENA region, political changes for example, have had some major implications on the MSL job profile and productivity while others, have had minimal effects.

\section{- Broad Problem Statement Research Objectives}

The research objective is to explain the relation between the tracking/reporting system for MSLs in medical affairs departments in pharmaceutical companies located within the MENA region and its impact on the MSL productivity therefore impacting the growth rate of the company taking in consideration the varying usage of the systems, frequency of tracking \& choice of metrics trying to prove the direct relation between all the variables.

\section{Major Research Question}

Does the use of tracking and reporting systems for pharmaceutical companies' MSLs increase their productivity?

Claim: The correct usage of these systems including correct metrics for pharmaceutical companies MSLs significantly increases their productivity.

\section{Minor Research Questions}

1. Does the use of tracking and reporting systems for pharmaceutical companies' MSLs impact their motivation?

Claim: There is a direct positive relation between correct usage of these systems including correct metrics for pharmaceutical companies MSLs and MSL motivation.

2. What metrics are considered imperative to capture that adequately measure MSL performance? Should the metrics contain quantitative \& qualitative metrics?

Claim: There is a direct positive relation between correct choice of metrics of the systems for pharmaceutical companies' MSLs and MSL productivity.

3. How frequently should these metrics be measured?

Claim: There is a direct positive relation between frequency of usage of the systems 
for pharmaceutical companies' MSLs and MSL productivity.

4. Should these tracking reports be readily available to internal stake holders or not? Claim: There is a direct positive relation between reports of the systems for pharmaceutical companies' MSLs being made readily available to internals take holders and MSL productivity.

5. Should the systems be job specific to the MSL role profile generally, or should they be customized to a company or therapy-area level?

6. Claim: There is a direct positive relation between customization of the systems for pharmaceutical companies MSLs and MSL productivity.

7. Will the use of these organizing tools help in better planning?

8. Claim: usage of the systems for pharmaceutical companies' MSLs enable better planning.

9. Can these reports be used as a tool in the MSL performance management process? Claim: usage of the systems for pharmaceutical companies' MSLs can be used as an effective tool in the end-of-year performance management process.

\section{Research Assumptions \&Limitations}

Assumption 1: Research is assuming that working conditions \& legalities are similar across countries of the MENA Region.

Limit 1: Limited to MENA Region countries as applied research.

Limit 2: The theoretical framework is limited to the variables included in the model.

Limit 3: Limited to the period 2010 - 2019.

\section{Literature Review}

Through searching Google Scholar® search engine on Saturday 17th February 2018 with the key words: "Medical-Science-Liaison reporting systems", a total of 299 articles were found. These were sorted according to date \& relevance to come up with the most suitable literature to include in this research. Twenty two articles were related to Medical-Science-Liaisons and were further read for relevance.

\section{Adding more keywords supported by literature / Relevant papers}

In an invention patent by (USA Patent No. $10 / 379,227$, 2003) regarding a method for outcome-based management of MSLs states that there is a need for a system to optimize the management of an MSL team and establish business metrics (measuring elements). This supports the major question of this research paper.

In another invention patent by (USA Patent No. $11 / 279,516,2006)$ regarding an apparatus for collecting data relating to MSLs which highlights on different generated reports that could be gained from MSL field activities. The author further this year went on to enhance the invention with fields that accepts voice input to reporting systems which he assumes enhances the MSL compliance to reporting.

(Chin, 2007) in this first of its kind article was very useful as he suggested that strategies (and hence metrics) may vary from company to company as well as customizing metrics per program which vary across the product lifecycle. The author emphasized that correct metrics would capitalize on outcomes and compliance.

Similar to (Chicharro, 2017), this was also highlighted by (Morris, 2009) in his article that "Differences in Perceived Role of Medical Affairs" between medical and commercial colleagues, "Medical Affairs and Brand Messaging Not Aligned" as well as "Suboptimal MSL Utilization" followed by the "Need to Demonstrate Value" are all solved by increased collaboration with commercial colleagues through planning and alignment as well as refined value metrics and accountability tracking necessary to developing a format for reporting accomplishments to senior management will be essential to demonstrate value. 
The multiple functions carried out by a medical affairs department vary in their emphasis across the life-cycle of a product (Ehrlich, 2013). This in turn supports our moderating variable two.

Effective and regular communication with internal stakeholders is critical for a successful global medical affairs department (Ehrlich, 2013). This supports our research in that moderating variable one along with independent variable two has a positive effect on MSL productivity. This has also been highlighted by (Wolin, 2001) in their article.

Due to the dynamic world of business within pharmaceutical companies nowadays and commercial executives measuring value in black-and-white terms, MSLs have to prove their value in the business cycle to internal stakeholders outside of the medical affairs function through submitting regular reports of trackable metrics. According to primary intelligence provider Cutting Edge Information, upper management are intensifying their pressure to quantify MSL value. This was highlighted in detail by (MSL Teams Regularly Track 10 or More KPIs, 2015) digging deeper into KPIs that MSL teams can benchmark performance against as well as best practices that top pharmaceutical companies use to demonstrate liaison value to internal stakeholders.

A survey was created by (Chicharro, 2017) for MSLs in the pharmaceutical industry in Spain and besides having the same problem of communicating MSL value to business partners, they had a difficult time demarking their roles from commercial colleagues in the company. This supports our moderating variable one.

\section{Comprehending the analyzed papers}

After careful reviewing of the relative literature, we can draw the following conclusions:

1. The need to establish business metrics (measuring elements).

2. Importance of generated reports to internal stake holders and enhancing MSL compliance by simplifying entry (for example using voice recognition).

3. Importance of increasing collaboration between medical and commercial colleagues to better demonstrate value of the MSL function which is sometimes underestimated.

4. Stating clearly that MSLs of different products in different stages of the product life- cycle carry out different jobs. This in turn supports customization of strategies (and hence metrics) at company and/or therapy area level.

5. Effective and regular communication and coordination with internal stake holders is critical for a successful global medical affairs department.

6. The need to close the gap between medical affairs quantitative nature of work and senior organization management with qualitative, tractable metrics analysis.

All of these previous conclusions support the conceptual research model and that there is a positive directional relationship between the variables initially suggested by our research paper.

\section{Hypothesis}

There could be a positive relation between the correct and efficient use of tracking and reporting systems for MSLs in medical affairs departments in MENA Region pharmaceutical companies and its impact on the MSL productivity and hence the growth rate of these companies taking in consideration that these reporting systems should be built on established business metrics that are quantitative and qualitative as well as being customizable per company or therapy area, taking in consideration the various jobs done by MSLs on different products in different stages of the product life-cycle.

Generated reports from these MSL tracking and reporting systems should be shared effectively and regularly within the organization to internal stakeholders, like commercial colleagues for 
example, which might increase collaboration and better demonstrate value of the MSL function which is critical.

$\mathrm{H}_{\mathbf{0}}$ 1: The use of tracking and reporting systems for MSLs of pharmaceutical companies along with the correct frequency increases productivity.

$\mathrm{H}_{\mathrm{A}}$ 1: The use of tracking and reporting systems for MSLs of pharmaceutical companies along with the correct frequency might increase productivity.

$\mathbf{H}_{\mathbf{0}}$ 2: The correct choice of metrics to track and report will increase productivity.

$\mathbf{H}_{\mathrm{A}}$ 2: The correct choice of metrics to track and report could increase productivity.

$\mathbf{H}_{0}$ 3: The ease of use of tracking and reporting systems with methods to increase compliance of users to the system (voice recognition for example) will increase productivity.

$\mathbf{H}_{\mathrm{A}}$ 3: The ease of use of tracking and reporting systems with methods to increase compliance of users to the system (voice recognition for example) might increase productivity.

$\mathrm{H}_{0} 4$ : Generated reports of the tracking and reporting systems for MSLs of pharmaceutical companies being made readily available to internal stakeholders (like commercial colleagues) increases productivity.

$\mathbf{H}_{\mathbf{A}}$ 4: Generated reports of the tracking and reporting systems for MSLs of pharmaceutical companies being made readily available to internal stakeholders (like commercial colleagues) could probably increase productivity.

$\mathbf{H}_{0}$ 5: The customization of metrics to company or therapy area level as well as differing per product as per its stage in the product life-cycle will increase productivity.

$\mathbf{H}_{\mathrm{A}}$ 5: The customization of metrics to company or therapy area level as well as differing per product as per its stage in the product life-cycle might increase productivity.

\section{Theoretical Framework \& Research Design \\ Theoretical Framework}

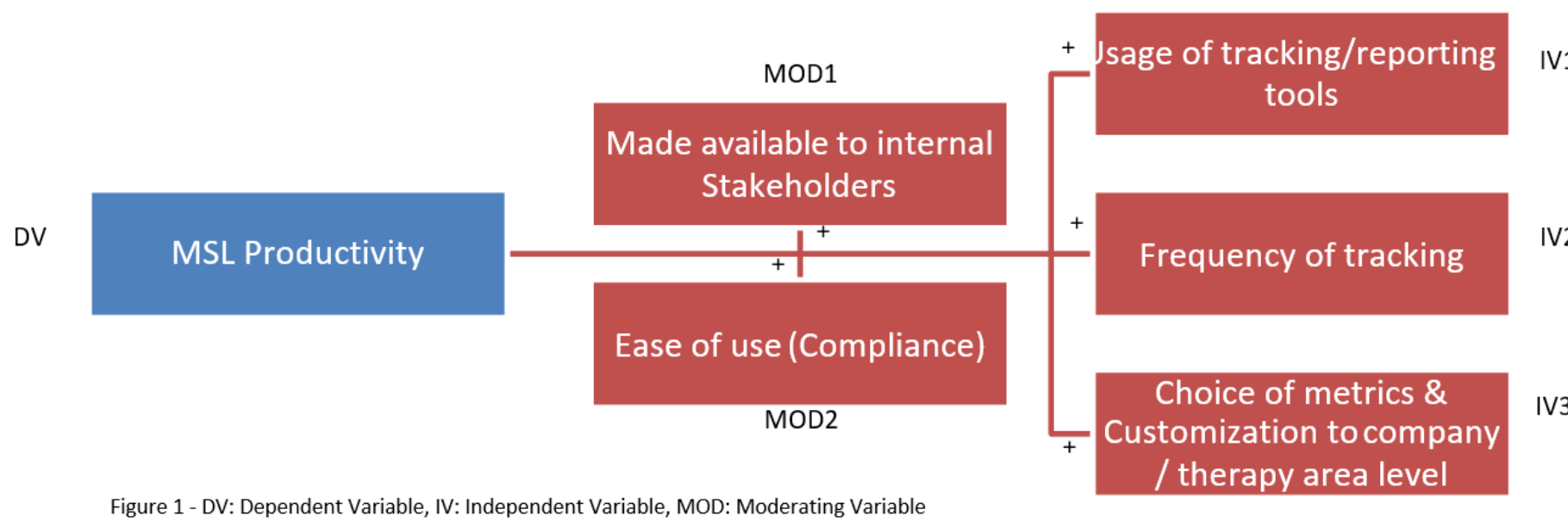

Figure 1: Suggested Theoretical Framework

\section{Research Variables}

- Dependent Variable

MSL Productivity: Productivity is an economic measure of output per unit of input.
In this context, it is the efficiency of the MSL in converting inputs (time \& effort along with tools) into meaningful outputs (feedback, insights, work activities \& associations). 


\section{- Independent Variable}

Usage of tracking/reporting tools with correct frequency: This refers to the correct and timely usage of the incorporated systems as per the internal guidelines.

Frequency of tracking: Refers to the number of times that the MSL has to fill/feed inputs to the system as well as the time interval that they need to spend doing so.

\section{Choice of metric \& Customization to} company/therapy area level: Which quantitative \& qualitative metrics combination is the best to accurately measure the MSL productivity and provide effective information to internal stakeholders? Will a generic system be sufficient to all MSLs? Or should they be customized to company and therapy area level so that MSLs of different therapy areas fill different reports?

\section{- Moderating Variable}

Made available to stakeholders: Making MSL reports readily available to internal medical and commercial colleagues will serve to increase MSL productivity.

Ease of use: Incorporation of technological advances like voice recognition or automation to further ease the use of the reporting systems and increase compliance.

\section{Research Methodology}

This research will be an exploratory research to test a non-directional hypothesis in my setting. The type of investigation is a descriptive analysis, correlation which will be noncontrolled (amount of control will be minimal $(0-20 \%))$ as it will deal with variables as they are without change.

The setting will be a field study and the time horizon will be cross-sectional as the data will be gathered once with regards to the theoretical framework highlighted above.

Data collection will be done through an electronic questionnaire developed through Free-online Surveys ${ }^{\circledR}$ to be distributed to a sample of 73 MSLs from the population of
MSLs in pharmaceutical companies in the MENA Region (calculated through sample size calculator at

http://www.raosoft.com/samplesize.html)

\section{Research Survey}

The electronic questionnaire distributed during February 2019 included - ensuring no biased questions and logical answer choices using a mixture of closed-ended as well as scale questions:

1. What is your gender?

2. Are you currently working as an MSL? or were you previously an MSL?

3. What Therapy Area do you manage?

4. What Country do you operate in?

5. Do you work for a Local or a MultiNational Pharmaceutical Company?

6. Does your company have a Tracking and/or Reporting System for MSLs?

7. How many years of experience do you have as an MSL?

8. What is the type of Tracking and/or Reporting System that your company uses?

9. What type of metrics does your company's Tracking and/or Reporting System capture?

10. Are the captured metrics stated above job specific to the MSL role profile generally, or are they customized to a company or therapy area level?

11. How frequently are those metrics measured and/or evaluated?

12. Do you use this Tracking and/or reporting System as a tool for planning?

13. What is the average percentage weight or impact of metrics captured by your company's system in your annual performance management review (Appraisal)?

14. From your personal judgement, are the captured metrics in the system adequate to measure MSL Performance? (Zero is not adequate at all \& Tenis very adequate) 
15. How frequently do you think these metrics should be measured and/or evaluated?

16. If your company's Tracking and/or Reporting System had a planning tool or function, would you use it to assist you in effectively planning your work?

17. In your personal judgement, do you think the percent age weight of metrics in your year- end performance review is an appropriate one? (Zero is not appropriate at all \& Ten is very appropriate)

18. MSL performance reports generated by the Tracking and/or Reporting System, should be readily made available to cross-functional stake holders to enhance productivity?

19. Does the use of Tracking and/or Reporting Systems for pharmaceutical companies' MSLs increase their productivity?

20. Does the use of Tracking and/or Reporting Systems for pharmaceutical companies' MSLs Negatively impact their motivation?

\section{Research Results}

\section{Survey Results}

The list of 73 targeted respondents was made up of $53 \%$ Male and $47 \%$ Female. $37 \%$ of the respondents were currently working as MSLs while the remaining $63 \%$ previously worked as MSLs.

The majority of respondents, exactly $71 \%$, worked in specialty care while $30 \%$ worked in primary care and only $14 \%$ worked in oncology. The responses were covering targeted respondents from MENA Region countries and the highest three countries were Egypt with $62 \%$, Saudi Arabia with 14\%, United Arab Emirates with $8 \%$. Nearly all of the respondents, 96\%, worked for multinational companies.

Out of the surveyed respondents $79 \%$ of them stated their companies had a tracking and reporting system in place during their working periods as MSLs. The majority - 78\% - of these systems were web-based systems.

Metrics captured by these systems were $90 \%$ a mixture of quantitative as well as qualitative metrics while only $10 \%$ of the systems captured quantitative metrics only. Moving onto customization of these metrics, $43 \%$ were company customized, $33 \%$ were general or generic metrics and 29\% were therapy area customized. The tracking of these metrics was done monthly in $67 \%$ of the cases.

The impact of the measured metrics within the associates' annual appraisal was for $34 \%$ of the responses in the average range (up to $30 \%$ ) while $31 \%$ of the responses were in the significant range (more than 50\%).

Coming to the personal judgement parts of the survey and on a scale from zero to ten, $33 \%$ of respondents think that the captured metrics in the system are adequate to measure MSL performance deserves 5 out of $10,17 \%$ gave a score of $7,12 \%$ gave a score of 8 .

When asked about their personal opinions on how frequently these MSL performance metrics should be measured, 52\% thought it should be measured quarterly, $36 \%$ monthly.

Personal opinions on a scale from zero to ten regarding the appropriateness of the impact of the measured metrics within the associates' annual appraisal were mid-range with numbers $5,6 \& 7$ each getting $19 \%$ of the responses.

Moving onto the planning aspect of the systems, $47 \%$ of surveyed MSLs use the systems as planning tools for their work, $26 \%$ do not use the system for planning although it does have thisfunctionality.

Asking the question in a different context for further clarifications, if the system had a planning function incorporated in it, $48 \%$ would use it for planning their work and $41 \%$ would maybe use it according to other circumstances in their opinion.

33\% agreed that MSL performance reports generated by the systems, should be readily made available to cross-functional stakeholders 
to enhance productivity, $14 \%$ strongly agree, $21 \%$ of the respondents disagreed and equally $21 \%$ were neutral while $12 \%$ strongly disagreed to this concept.

Coming to one of the main focus questions of this research it was found that $47 \%$ agreed that the use of these systems increased their productivity, 28\% strongly agreed, $21 \%$ were neutral towards this and only $3 \%$ disagreed and $2 \%$ strongly disagreed.

Upon being asked if these systems negatively impact their motivation, 29\% disagreed while $22 \%$ agreed and $26 \%$ were neutral.

The full detailed survey results are available in Annex 1 at the end of this research paper.

\section{Discussion}

The list of 73 targeted respondents initially calculated as the representative sample was reached after 6 days of launching the survey online during February 2019 and using specialized social media platforms like LinkedIn ${ }^{\circledR}$ to professionally promote for the survey to targeted audience as well as face-toface interactions in Egypt \& Saudi Arabia.

All respondents were from the intended targeted audience and well balanced between genders with $53 \%$ Male and $47 \%$ Female. $37 \%$ of the respondents were currently working as MSLs while the remaining $63 \%$ previously worked as MSLs dating back to 2010.

With a slight overlap for associates that worked for multiple therapy areas during their MSL period, most respondents managed two therapy areas during their period working as an MSL. The majority of respondents reaching over $70 \%$ managed specialty care while the rest was a mixture of primary care and oncology therapy areas.

The top represented country in our research was Egypt followed by Saudi Arabia then United Arab Emirates with the rest of the main countries in the MENA Region all coming in following position with similar representation amongst the countries. This may be a slight representation of bias since these countries had face-to-face briefing of respondents prior to taking the survey while other countries lacked this. Another limitation of this research related to geography was the lack of representation from other major countries in the MENA Region like Turkey \& Iran.

Nearly all surveyed respondents worked for multinational companies which emphasizes on the global direction of the incorporation of the systems in MSL performance evaluation and it was not surprising to find that the majority of these companies had tracking and reporting systems already in place. These available systems were mainly web-based CRM systems in the majority of cases and nearly all of them tracked a mixture of qualitative and quantitative metrics for MSL performance.

With regards to the in-depth analysis of the MSL performance metrics captured, previously mentioned to be a mixture of qualitative \& quantitative metrics, there was a clear overlap between company customized metrics that related specific MSL performance aspects to tailored company approaches and general or generic MSL metrics like number of HCPs covered or number of visits for example where both contributed $43 \%$ and $33 \%$ respectively. Results conclude having the correct mixture of quantitative and qualitative metrics enhances performance.

These tracked metrics were mainly tracked monthly in the majority of companies while overlapped with either a quarterly or annual review of performance in most companies. The main aspect that the majority of respondents wanted to change is the time of tracking where over half of the respondents preferred quarterly tracking followed by monthly. The results suggest there seems to be a direct positive relation between frequency of usage of the systems and MSL productivity.

To ensure better optimized performance for MSLs, the systems can be used as effective tools in the end-of-year performance management process. In over half of the surveyed respondents, current tracked metrics 
make up around $30 \%$ to $50 \%$ impact of annual appraisal of MSLs.which shows a clear association in the eyes of pharmaceutical company executives that MSL performance metrics - regardless of type and tracking period - define the overall value of the MSL and hence their annual appraisal.

Being dynamic systems, nearly half of the respondents used the already available systems for planning of their work which clearly indicates the importance of these tools in having an effective and productive business cycle for MSLs. This is backed up by a fair number of respondents using other tools to plan their work, which signals the importance of planning for MSLs working in pharmaceutical companies across the MENA Region.

To conclude the discussion and to support the initial hypothesis, $75 \%$ of the respondents believe that the use of tracking and reporting systems will increase their productivity and nearly half of the respondents believe that making the generated MSL reports readily available for internal stakeholders will further enhance productivity both of which will not negatively impact motivation of MSLs in pharmaceutical companies operating the MENA Region. This is In line with the hypothesized direct positive relation and capitalizing on the importance of cross-functional collaboration.

\section{Conclusion}

The research paper at hand has proven that there is a positive relation between the correct and efficient use of tracking and reporting systems for MSLs in medical affairs departments in MENA Region pharmaceutical companies and its impact on the MSL productivity and hence the growth rate of these companies taking in consideration that these reporting systems should be built on established business metrics that are quantitative and qualitative as well as being customizable per company or therapy area, taking in consideration the various jobs done by MSLs on different products in different stages of the product life-cycle.

Generated reports from these MSL tracking and reporting systems should be shared effectively and regularly within the organization to internal stakeholders to increase collaboration and better demonstrate value of the MSL function.

\section{Bibliography}

1. Albert, E. a. (2011). The Medical Science Liaison: An A to $Z$ Guide, Second Edition" (Second ed., Vol. 10). eBookIt.com. doi:978-1-4566-0430-1

2. Almeida. (2014). The "emerging" role of Medical Science Liaison in pharmaceutical industry. Repositório Institucional da Universidade de Aveiro. Retrieved from https://ria.ua.pt/handle/10773/13603

3. Baptista. (2006, April 12). USA Patent No. 11/279,516. doi:US 2007/0244715 A1

4. Bass, J. L. (2009, November 1). Surveys of Medical Liaison Practices across the Pharmaceutical Industry: A Review. Sage Journals, 43(6), 685-695. doi:https://doi.org/10.1177/0092861509 04300606

5. Batista. (2006, April 12). USA Patent No. 11/279,525. doi:US 2007/0244717 A1

6. Bhambri, R. (2014, October 27). Working Outside the Dispensing Box: The Roles of the Medical Information Specialist and Medical Science Liaison. Pharmacy Times, pp. 1-2. Retrieved February 19, 2018, from http://www.pharmacytimes.com/publicatio ns/career/2014/pharmacycareers_fall2014/ working-outside--the-dispensing-boxthe-roles-of-the-medical-informationspecialist-and- medical-science-liaison

7. Bijker, M. (2017, July 22). IgeaHub. Retrieved February 10, 2018, from Career, Prespectives: https://igeahub.com/2017/07/22/medical -science-liaison-msl-all-you-need-to- 
know/

8. Chicharro, A. (2017, July 27). A survey of medical scientific liaisons in the pharmaceutical industry in Spain. Journal of Medical Marketing: Device, Diagnostic and Pharmaceutical Marketing, $\quad$ 16(1), 4-9. doi:https://doi.org/10.1177/1745790417 717086

9. Chin, J. (2007). Measuring performance of field-medical programmes: Medical science liaison metrics consensus. Journal of Commercial Biotechnology, 13(1), $\quad 177 \quad$ - 182. doi:10.1057/palgrave.jcb.3050057.

10. Cutting Edge Information. (2015, November 5). Retrieved February 10, 2018, from Market Wired: http://www.marketwired.com/pressrelease/medical-science-liaison-teamsregularly-track-10-or-more-keyperformance-indicators-2070807.htm

11. Cutting Edge Information. (2016, February 23). Retrieved February 10, 2018, from Market Wired: http://www.marketwired.com/pressrelease/key-performance-indicatorstracked-surveyed-medical-scienceliaison-teams-united-states-2099402.htm 12. Ehrlich, H. (2013). The Emerging Role of Global Medical Affairs in the Twenty-First Centruy. Production of Plasma Protein for Therapeutic Use(First Edition), 413 - 421. Retrieved February 19, 2018, from https://books.google.com.eg/books?hl=e n\&lr=\&id=MGLOQOc-

rtsC\&oi=fnd\&pg=PA413\&dq=\%22Med ical-Science-

Liaison\%22++reporting+systems\&ots=r6g g83WzkX\&sig=VJtiu7Ky9Twt7LWsxU91 Mo

E90EQ\&redir_esc $=\mathrm{y} \# \mathrm{v}=$ onepage $\& \mathrm{q} \& \mathrm{f}=$ false

13. Firth, N. (2000, October 1). Outsourcing Industry Medical Information Services.
Therapeutic Innovation \& Regulatory Science (TIRS), 34(4), 1105-1113. doi: https://doi.org/10.1177/00928615000340 0416.

14. Giffin, S. A. (2014, October 30). Review of the Evolution of Medical Information in Light of Changes in the External Landscape. Therapeutic Innovation \& Regulatory Science (TIRS), 48(6), NP6NP14. doi:doi.org/10.1177/2168479014547935

15. Kulkarni, D. (2015, July 24). USA Patent No. 14/808,744. doi:US 2016/0027023 A1 Massey. (2003, March 4). USA Patent No. 10/379,227. doi:US 2004/0177071 A1.

16. Meyer, J. O. (2015, May 1). PhD Thesis Defence.

Empowerment, transformational leadership, and intention to quit in a medical science liaison team. WALSH, USA: Pro Quest LLC.

17. Morgan, D. K. (2000, October 1). History and Evolution of Field-Based Medical Programs. Therapeutic Innovation \& Regulatory Science (TIRS), pp. 1049-1052. doi:10.1177/009286150003400408.

18. Morris, T. D. (2009). Optimizing the Impact of the Medical Affairs Function. Campbell Alliance, 1-5. Retrieved February 19, 2018, from http://www.prnresource.com/wpcontent/uploads/2009/08/medical_affairs _functionoptimizing.pdf.

19. Rutherford, P. (2012, September). Managing MSLs In A Global Medical Organization: Budget, Staffing And Compensation Benchmarks. Cutting Edge Information. Retrieved from https://www.iqvia.com/-

/media/library/white-papers/medicalscience-liaisons.pdf

20. Smith, N. J. (2015, October). The MSL KPI Disconnect: A Global Survey of MSL KPIs and Metrics. The Medical 
Science Liaison Society. Retrieved from https://www.iqvia.com//media/library/white-papers/medicalscience-liaisons.pdf

21. Werling, K. (2011, November 1). Focus on Life Science Compliance: The Evolution of Medical Affairs Departments. AHLA Connections, 14 18. Retrieved February 19, 2018, from https://www.mcguirewoods.com/newsresources/publications/health_care/focus -life- science-compliance-nov-2011.pdf

22. Wolin, M. J. (2001, April 1). The Emerging Role of Medical Affairs within the Modern Pharmaceutical Company. 35(2), 547-555. doi: https://doi.org/10.1177/00928615010350 022

\section{List of Abbreviations}

1. MSL: Medical Science Liaison

2. KOL: Key Opinion Leader - broadly defined as the clinical leaders in their disease area

3. MENA: Middle East \& North Africa Region

4. HCPs: Health Care Professionals

5. IIT: Investigator Initiated Trial

6. CRM: Customer Relationship Management

7. IT: Information Technology

8. KPI: Key Performance Indicators

9. HR: Human Resources 
Annex 1

Introduction

2 Are you currently working as an MSL? or were you previously an MSL?

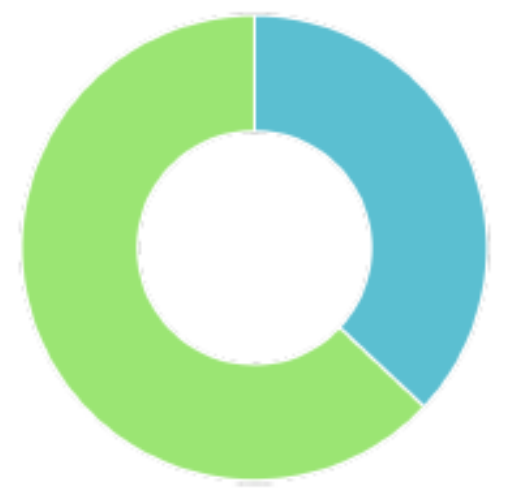

Standard Deviation
$37 \%(27)$

Currently Working as MSL

9.5

Standard Deviation
1 What is your gender?

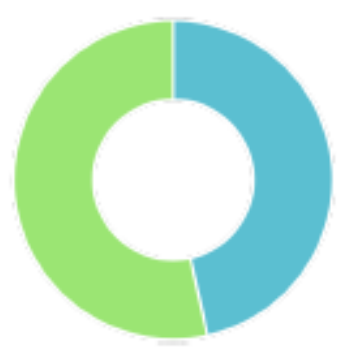

$47 \%(34)$

Female

$53 \%(39)$

Male

2.5

73

Responses
$63 \%(46)$

Previously Working as MSL

73

Responses 
3 What Therapy Area do you manage?

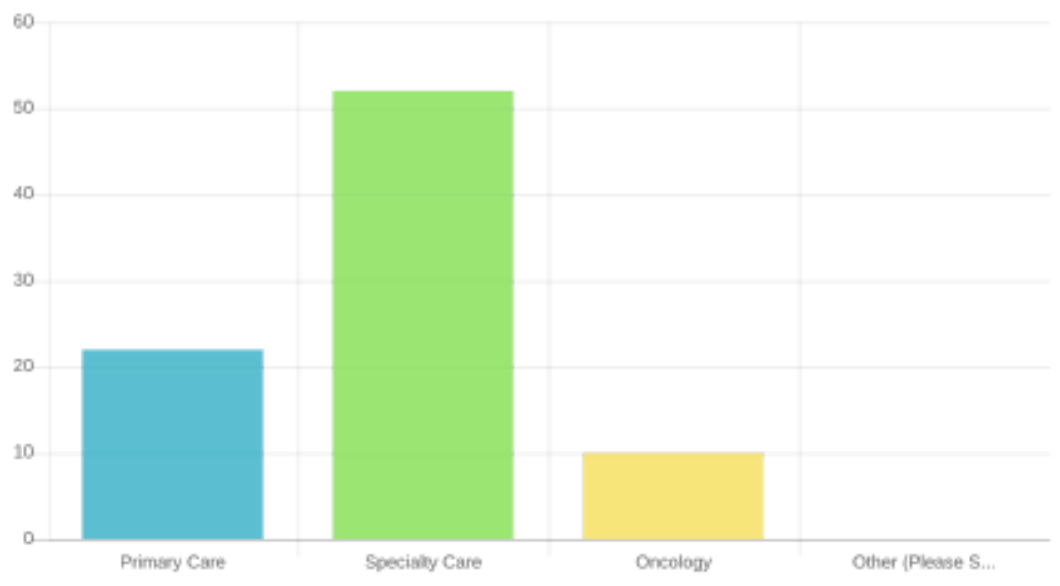

$30 \%(22)$

Primary Care

73

Responses

\section{$71 \%(52)$}

Specialty Care
$14 \%(10)$

Oncology
$0 \%(0)$

Other (Please Specify)

4 What Country do you operate in?

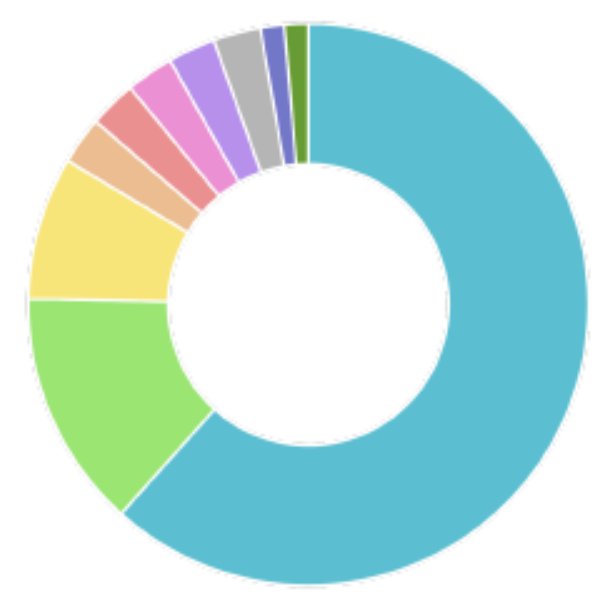

\begin{tabular}{|c|c|c|c|}
\hline $62 \%(45)$ & $14 \%(10)$ & $8 \%(6)$ & $3 \%(2)$ \\
\hline Egypt & Saudi Arabia & United Arab Emirates & Iran \\
\hline 3\%(2) & $3 \%(2)$ & $3 \%(2)$ & $3 \%(2)$ \\
\hline Jordan & Lebanon & North Sudan & South Sudar \\
\hline 1\%(1) & $1 \%(1)$ & $0 \%(0)$ & $0 \%(0)$ \\
\hline Tunisia & Other (Please Specify) & Algeria & Bahrain \\
\hline $0 \%(0)$ & $0 \%(0)$ & $0 \%(0)$ & $0 \%$ \\
\hline Iraq & Kuwait & Libya & Other \\
\hline
\end{tabular}

10.75

Standard Deviation

73

Responses 
7 How many years of experience do you have as an MSL?

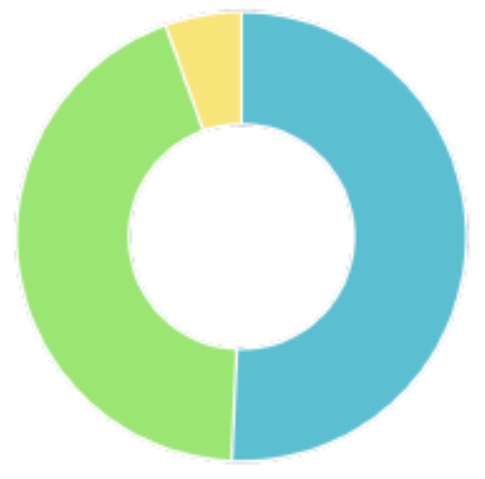

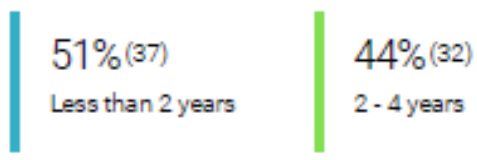

$5 \%(4)$

4- 6 years

$0 \%(0)$

More than 6 Years

16.41
Standard Deviation

73

Responses

\section{Tracking \& Reporting System Overview}

8 What is the type of Tracking and/or Reporting System that your company uses?
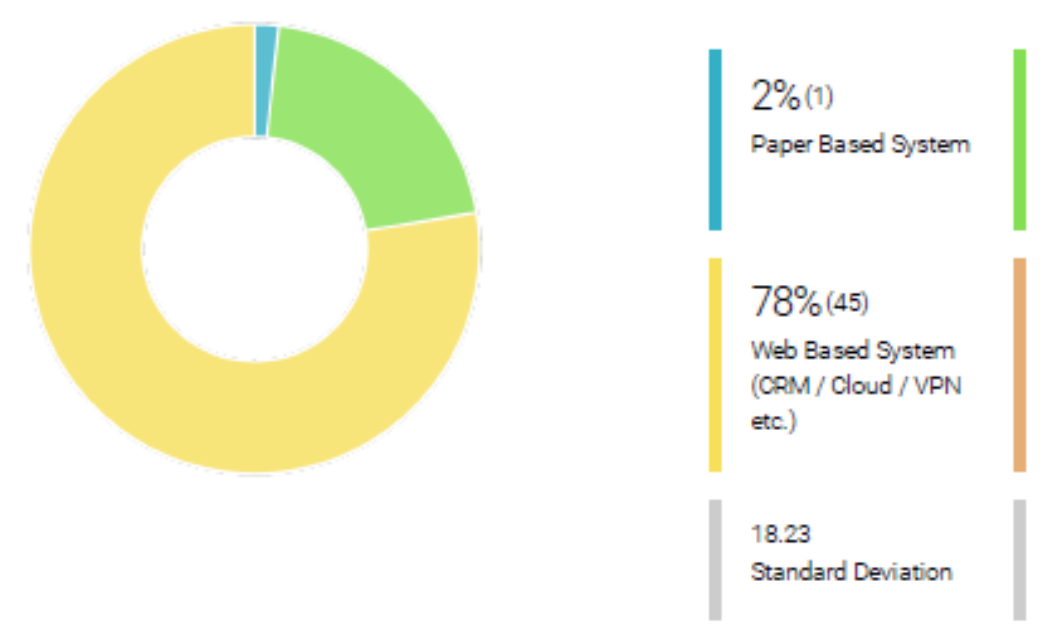

$21 \%(12)$

Local Laptop Based System

\section{$0 \%(0)$}

Other (Please Specify)

58

Responses

9 What type of metrics does your company's Tracking and/or Reporting System capture?

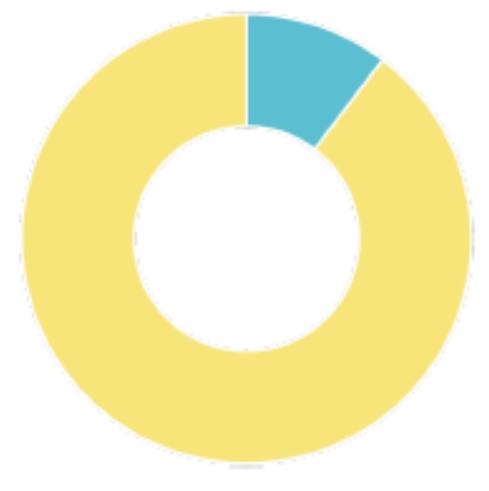

\begin{tabular}{|c|c|}
\hline $10 \%(6)$ & $0 \%(0)$ \\
\hline $\begin{array}{l}\text { Quantitative Metrics } \\
\text { Only }\end{array}$ & $\begin{array}{l}\text { Qualitative Metrics } \\
\text { Only }\end{array}$ \\
\hline $90 \%(52)$ & $0 \%(0)$ \\
\hline $\begin{array}{l}\text { Quantitative \& } \\
\text { Qualitative Metrics }\end{array}$ & Other (Please Specify) \\
\hline 21.79 & 58 \\
\hline Standard Deviation & Responses \\
\hline
\end{tabular}


10 Are the captured metrics stated above job specific to the MSL role profile generally, or are they customized to a company or therapy area level?

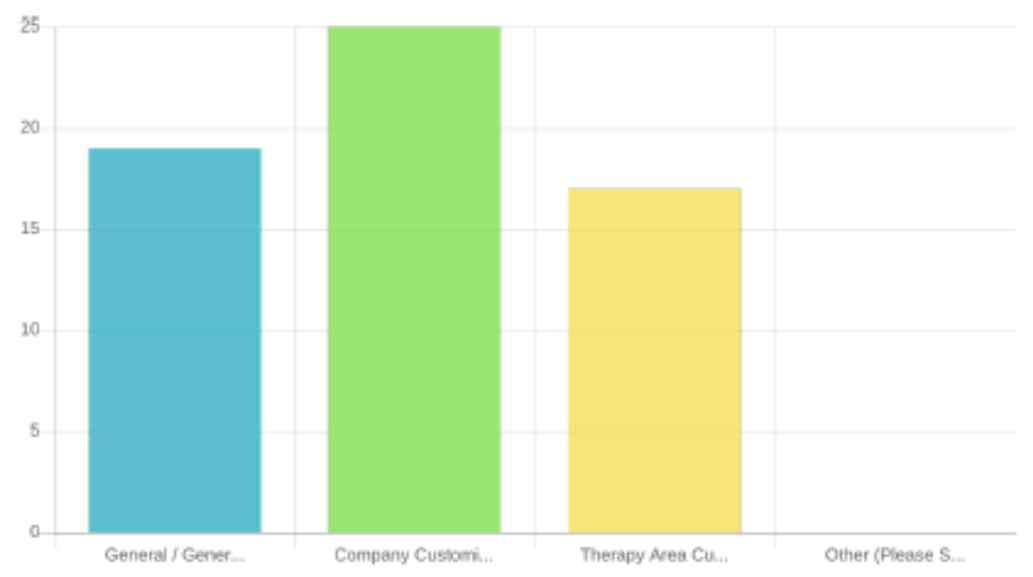

\begin{tabular}{l|l|l||l}
$33 \%(19)$ & $43 \%(25)$ & $29 \%(17)$ & $0 \%(0)$ \\
General / Generic Metrics & Company Customized & Therapy Area Customized & Other (Please Specify)
\end{tabular}

58

Responses

11 How frequently are those metrics measured and/or evaluated?

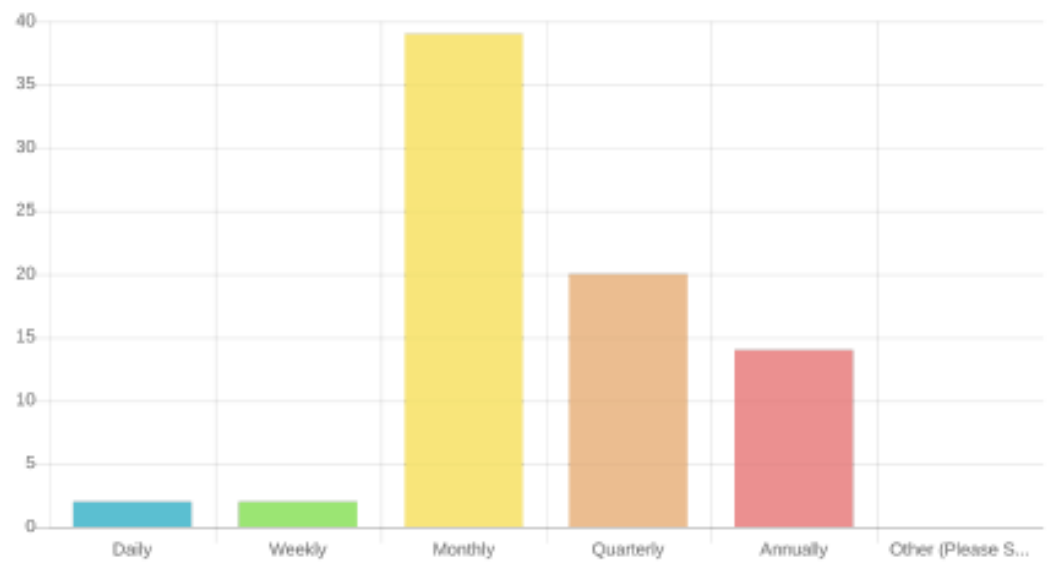

$3 \%(2)$

Daily

$24 \%(14)$

Annually

58

Responses

\section{$3 \%(2)$}

Weekly

$67 \%(39)$

Monthly

$0 \%(0)$

Other (Please Specify)
$34 \%(20)$

Quarterly 
12 Do you use this Tracking and/or reporting System as a tool for planning?

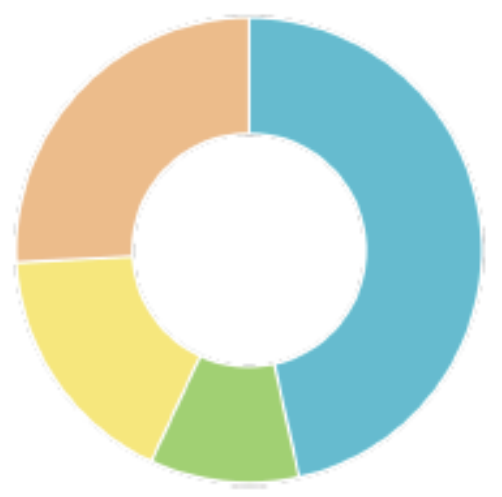

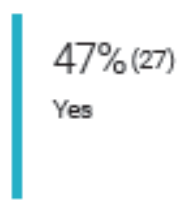

$17 \%$ (10)

No, I use other

planning tools

$0 \%(0)$

Other (Please Specify)

9.13

Standard Deviation
$10 \%(6)$

No, it doesn't have

this option

$26 \%(15)$

No, I donit use it for planning

58

Responses

13 What is the average percentage weight or impact of metrics captured by your company's system in your annual performance management review (Appraisal)?
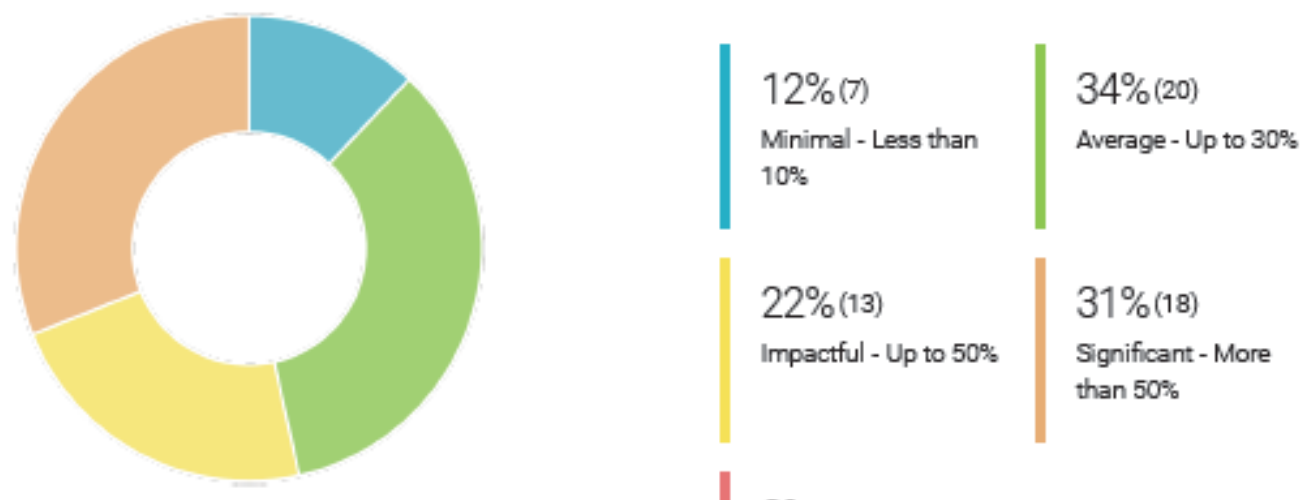

$0 \%(0)$

Other (Please Specify)

7.34

Standard Deviation

58

Responses 


\section{JMSCR Vol||08||Issue ||07||Page 274-292||July}

Tracking and/or Reporting Systems Evaluation

14 From your personal judgement, are the captured metrics in the system adequate to measure MSL Performance? (Zero is not adequate at all \& Ten is very adequate)

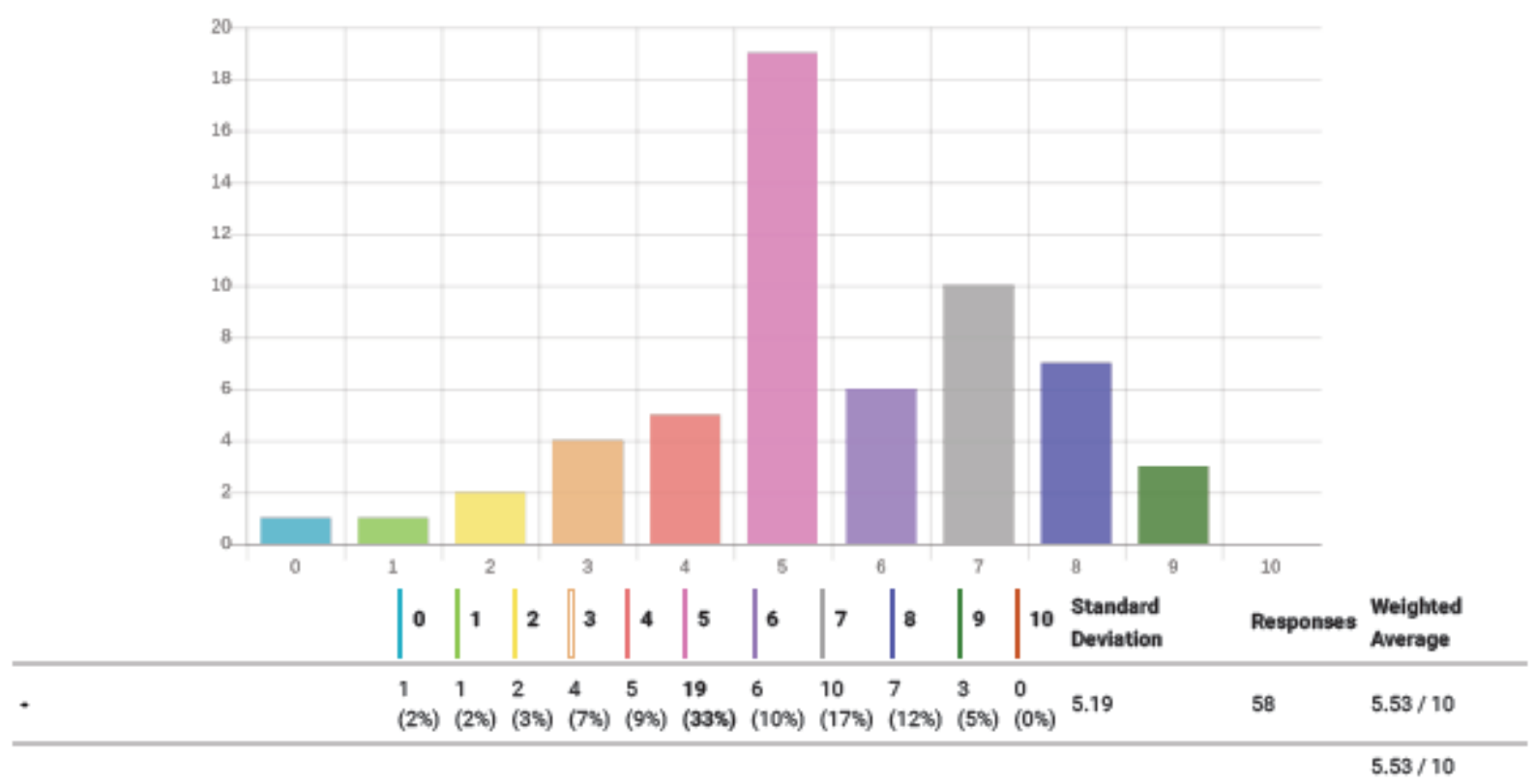

18 MSL performance reports generated by the Tracking and/or Reporting System, should be readily made available to cross-functional stakeholders to enhance productivity?

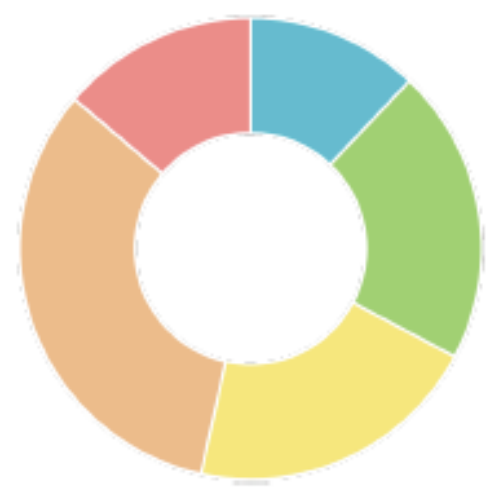

\begin{tabular}{|l|l}
\hline $\begin{array}{l}12 \%(7) \\
\text { Strongly Disagree }\end{array}$ & $\begin{array}{l}21 \%(12) \\
\text { Disagree }\end{array}$ \\
$\begin{array}{l}\text { 21\%(12) } \\
\text { Neutral }\end{array}$ & $\begin{array}{l}33 \%(19) \\
\text { Agree }\end{array}$ \\
& $\begin{array}{l}0 \%(0) \\
\text { Other (Please Specify) }\end{array}$ \\
\hline $\begin{array}{l}\text { Strongly Agree } \\
\text { (8) }\end{array}$ & $\begin{array}{l}58 \\
\text { Responses }\end{array}$ \\
\hline $\begin{array}{l}5.79 \\
\text { Standard Deviation }\end{array}$ &
\end{tabular}


19 Does the use of Tracking and/or Reporting Systems for pharmaceutical companies' MSLS increase their productivity?
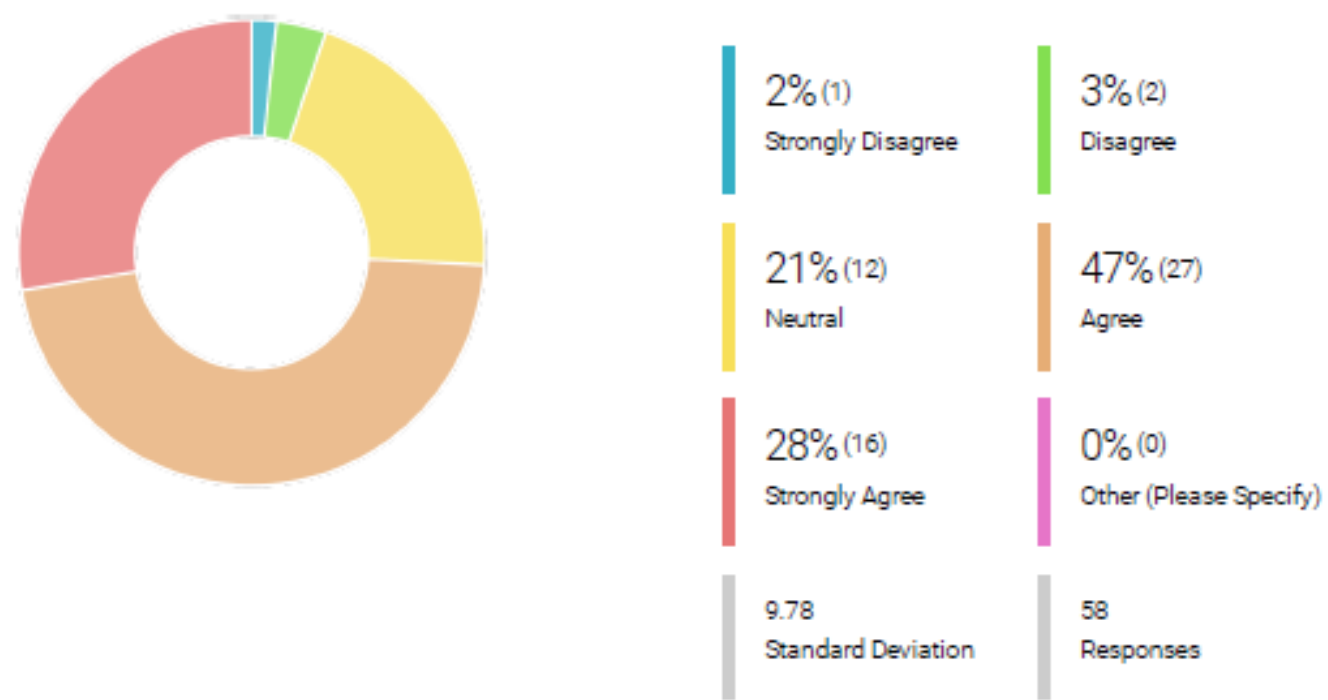

20 Does the use of Tracking and/or Reporting Systems for pharmaceutical companies' MSLs Negatively impact their motivation?
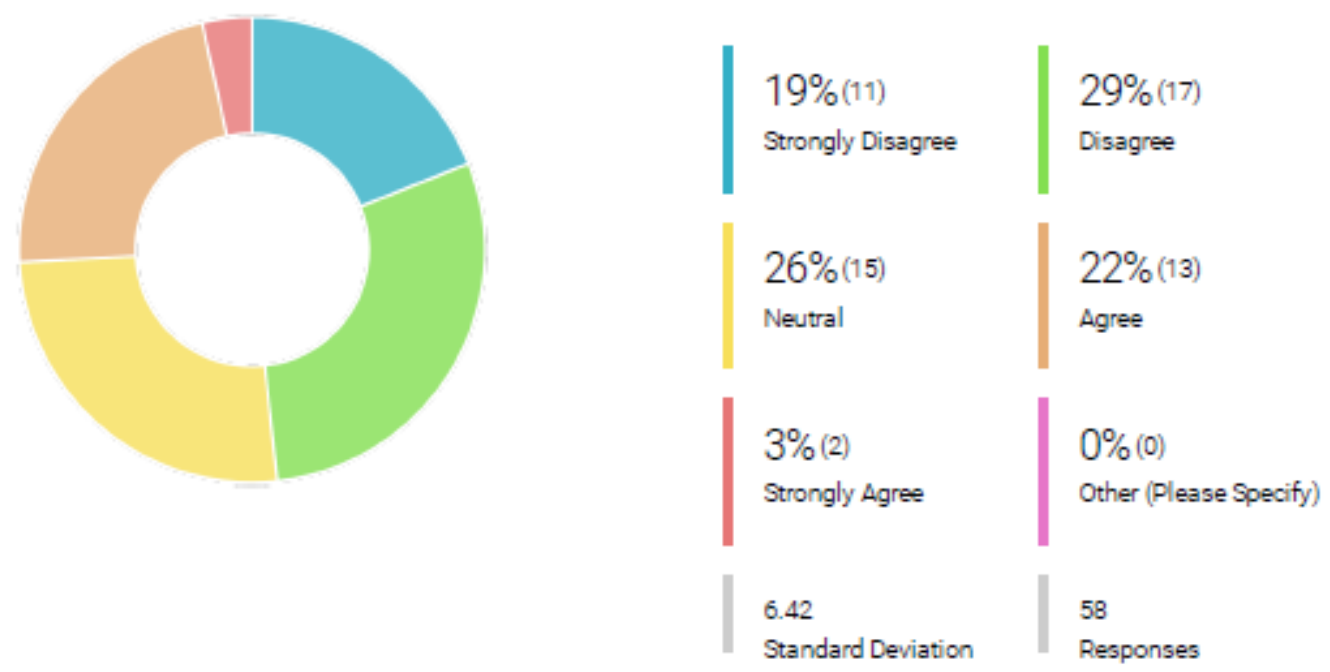